\title{
Big rational surfaces
}

\author{
Damiano Testa • Anthony Várilly-Alvarado • \\ Mauricio Velasco
}

Received: 4 March 2010 / Revised: 27 September 2010 / Published online: 11 November 2010 (C) The Author(s) 2010. This article is published with open access at Springerlink.com

\begin{abstract}
We prove that the Cox ring of a smooth rational surface with big anticanonical class is finitely generated. We classify surfaces of this type that are blow-ups of $\mathbb{P}^{2}$ at distinct points lying on a (possibly reducible) cubic.
\end{abstract}

Mathematics Subject Classification (2000) Primary 14J26; Secondary 14M05

\section{Introduction}

\subsection{Mori dream spaces}

Let $X$ be a smooth projective variety over an algebraically closed field. Assume that the Picard group Pic $(X)$ is freely generated by the classes of divisors $D_{1}, D_{2}, \ldots, D_{r}$. The Cox ring, or total coordinate ring, of $X$ with respect to this choice is given by

$$
\operatorname{Cox}(X):=\bigoplus_{\left(m_{1}, \ldots, m_{r}\right) \in \mathbb{Z}^{r}} \mathrm{H}^{0}\left(X, \mathcal{O}_{X}\left(m_{1} D_{1}+\cdots+m_{r} D_{r}\right)\right),
$$

with multiplication induced by product of functions in the function field of $X$.

D. Testa was partially supported by Jacobs University Bremen, DFG grant STO-299/4-1 and EPSRC grant number EP/F060661/1; the third author is partially supported by NSF grant DMS-0802851.

\section{Testa $(\varangle)$}

Mathematical Institute, 24-29 St Giles, Oxford OX1 3LB, United Kingdom

e-mail: adomani@gmail.com

A. Várilly-Alvarado

Department of Mathematics, MS 136, Rice University Houston, Houston, TX 77005, USA

e-mail: av15@rice.edu

M. Velasco

Department of Mathematics, University of California, Berkeley, CA 94720, USA

e-mail: mauvelasco@gmail.com 
The Cox rings of certain classes of varieties are particularly simple. In the case of toric varieties, for instance, the Cox ring is the ring of polynomial functions on an affine space $\mathbb{A}^{d}$ with coordinates indexed by the torus-invariant divisors [7, Theorem 2.1]. Moreover, the variety $X$ can be recovered as a quotient of an open subset of $\mathbb{A}^{d}=$ $\operatorname{Spec}(\operatorname{Cox}(X))$ by the action of a torus. More generally, any smooth projective variety $X$ with a finitely generated Cox ring can be described in this way: there is an open subset $\mathcal{T}$ of $\operatorname{Spec}(\operatorname{Cox}(X))$ with a canonical torus action and the quotient of $\mathcal{T}$ by this action is isomorphic to $X$. The space $\mathcal{T}$ is an example of a universal torsor (see [6] for a foundational treatment of universal torsors).

Varieties $X$ with finitely generated Cox ring are also distinguished amongst all varieties: the minimal model program on $X$ can be carried out for any divisor. This privileged position has earned such varieties the name of Mori dream spaces [17, Definition 1.10]. Determining which varieties are Mori dream spaces remains a difficult problem, even in the case of surfaces. In this paper, we are primarily interested in the case in which $X$ is a smooth rational surface with big anticanonical divisor. We prove the following result.

Theorem 1 Let $X$ be a smooth rational surface such that $-K_{X}$ is big. Then the Cox ring of $X$ is finitely generated.

This result extends a theorem of Hassett [16, Theorem 5.8], which states that a smooth rational surface with big and nef anticanonical divisor is a Mori dream space. Our generalization provides us with new examples of rational surfaces with finitely generated Cox ring whose Picard rank is arbitrarily large.

As a consequence of recent advances in the Minimal Model Program ([3]) it follows that every log-Fano variety is a Mori dream space. In the context of rational surfaces Theorem 1 implies this result and is properly more general (see Sects. 1.3, 3). A further advantage of our result is that the property of having big anticanonical divisor is a property that is closed under specialization in smooth proper families. One of the consequences of this feature lies in the applications to arithmetic: Cox rings have been used to study Manin's Conjecture on singular del Pezzo surfaces and our result provides a general framework for proving finite generation of Cox rings in this context.

As we were completing this paper, we learned that Chen and Schnell obtained an independent proof of Theorem 1.

To prove Theorem 1, we use a result of Galindo and Monserrat [11] which reduces the problem to showing that $X$ contains finitely many curves with negative selfintersection, and that a certain subring of $\operatorname{Cox}(X)$ is finitely generated. The first requirement follows from work of Sakai [25]; the second can in turn be reduced, by a lemma of Zariski, to showing that every nef divisor on $X$ is semiample. This final claim follows from a few classical facts. We prove Theorem 1 in Sect. 2; in some cases, we give elementary, self-contained proofs of the necessary reductions.

Example 1 We deduce from Theorem 1 that the surfaces in the following list are Mori dream spaces (see Remark 2).

a. Rational surfaces with $K_{X}^{2}>0$, or equivalently, rational surfaces with $\operatorname{rk}(\operatorname{Pic}(X)) \leq$ 9, are Mori dream spaces (see also [11, Corollary 1]). 
b. Blow-ups of the Hirzebruch surface $\mathbb{F}_{n}, n \geq 1$ at any number of points lying in the union of the negative curve and $n+1$ distinct fibers of the projection.

c. Blow-ups of $\mathbb{P}^{2}$ at $n+1$ points, $n$ of which lie on a (possibly reducible) conic (see also [11, Corollary 3] for the case in which the points are contained in a conic).

d. The surface obtained by blowing up $\mathbb{P}^{2}$ at the ten points of pairwise intersections of five general lines (Fig. 1). Generators for the Cox ring of this surface are determined in [4]. Harbourne and Roé have also shown that the surface in question is a Mori dream space ([14]).

e. The surface obtained by considering three distinct lines $L_{1}, L_{2}, L_{3}$ in $\mathbb{P}^{2}$ and blowing up the three pairwise intersections and 2, 3 and 5 additional points on $L_{1}, L_{2}$ and $L_{3}$ respectively.

In view of Theorem 1 it is natural to attempt a classification of big rational surfaces. We make a first step towards this goal by classifying blow-ups of $\mathbb{P}^{2}$ at finite sets of points for which $-K_{X}$ is both big and effective (Theorem 2). The classification is achieved by associating a root system to each big rational surface. There are many examples in the literature of root systems associated to rational surfaces (see, for instance, $[8,21,22])$; ours is a natural extension of the construction in [22].

Theorem 2 Let $\pi: X \rightarrow \mathbb{P}^{2}$ be the blow-up of $\mathbb{P}^{2}$ at $r$ distinct points. Then $-K_{X}$ is effective and big if and only if one of the following holds:

1. $r \leq 8$;

2. a general element of $\left|-K_{X}\right|$ consists of the strict transform of a line and a conic where exactly a of the blown-up points lie exclusively on the line, exactly $b$ of the blown-up points lie exclusively on the conic, and either $a b=0$ or $\frac{1}{a}+\frac{4}{b}>1$;

3. a general element of $\left|-K_{X}\right|$ consists of the strict transform of three lines $L_{1}, L_{2}$ and $L_{3}$ where for $i \in\{1,2,3\}$ exactly $a_{i}$ blown-up points lie exclusively on the line $L_{i}$, and either $a_{1} a_{2} a_{3}=0$ or $\frac{1}{a_{1}}+\frac{1}{a_{2}}+\frac{1}{a_{3}}>1$.

There are smooth projective rational surfaces with finitely generated Cox ring, whose anticanonical divisor is not big. For example, by [26, Theorem 5.2] the surface $X$ obtained by blowing up the nine inflection points of a smooth plane cubic has finitely generated Cox ring. However, the anticanonical divisor $-K_{X}$ is not big since $\left|-K_{X}\right|$ contains an irreducible curve and $K_{X}^{2}=0$.
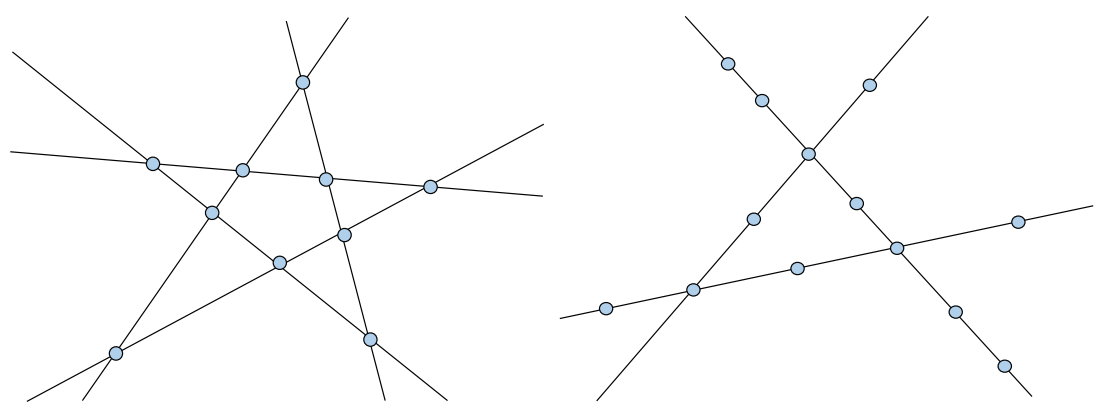

Fig. 1 Examples 1 (d) and (e) 
In the recent preprint [1], Artebani and Laface study the problem of finite generation of the Cox rings of surfaces with smaller anticanonical Iitaka dimension.

\subsection{Previous work}

Smooth rational surfaces with big anticanonical class have been studied before. Sakai showed that their anticanonical models have only isolated rational singularities and he provided numerous examples of them ([25]).

It is known that the effective cones of the surfaces appearing in Theorem 2 are finitely generated. This was shown for surfaces type (1) by Harbourne in [12] and by Harbourne, Geramita and Migliore in [15]. For surfaces of types (2) and (3) it was shown by Failla, Lahyane and Molica Bisci in $[9,10]$. In the case of the blow-up of $a$ points on a line (i.e. case (2) with $b=0$ ), Ottem obtained a presentation for the Cox ring ([24]). Theorem 2 unifies and strengthens these results.

\subsection{Relation to log del Pezzo surfaces}

A $\log$ del Pezzo surface (i.e., a Kawamata log terminal pair $(X, \Delta)$ such that $X$ is a normal surface and $-\left(K_{X}+\Delta\right)$ is $\mathbb{Q}$-Cartier and ample) is a Mori dream space [3, Corollary 1.3.2]. Clearly, log del Pezzo surfaces have big anticanonical class. It is natural to wonder if the converse is true. It is not. In Sect. 3 we give a family of examples, suggested to us by Chenyang $\mathrm{Xu}$, which shows that the class of smooth rational surfaces with big anticanonical class is strictly larger than the class of minimal resolutions of log del Pezzo surfaces.

\section{Big rational surfaces are Mori dream spaces}

Let $X$ be a smooth projective variety. Recall that a (real) divisor $B$ on $X$ is big if there are an ample divisor $A$ and an effective divisor $E$ on $X$ such that $B$ is numerically equivalent to $A+E$. An integral divisor $B$ on $X$ is semi-ample if there is a positive integer $n$ such that the linear system $|n B|$ is base point free. See [20, Chapter 2].

Notation. From now on, unless otherwise specified, $X$ denotes a smooth projective rational surface over an algebraically closed field with big anticanonical divisor $-K_{X}$. We note that $\operatorname{Pic}(X)$ is a free abelian group and that $\operatorname{rk}(\operatorname{Pic}(X))+$ $K_{X}^{2}=10$. Let $N_{1}(X)$ be the $\mathbb{R}$-vector space of numerical equivalence classes of curves on $X$; let $N E(X)$ be the cone in $N_{1}(X)$ of non-negative real combinations of classes of curves on $X$ and let $\overline{N E}(X)$ be its closure. We denote by $N E(X)_{\mathbb{Z}}$ the cone of non-negative integral linear combinations of classes of curves on $X$.

We begin with a short proof of a weaker version of [11, Theorem 1]; this result does not require $X$ to be a rational surface, and it reduces Theorem 1 to showing that $X$ contains finitely many curves with negative self-intersection, and that the "nef subring" of $\operatorname{Cox}(X)$ is finitely generated. 
Proposition 1 The Cox ring of $X$ is generated by global sections supported on the curves with negative self-intersection and generators of the subring $\bigoplus_{N \text { nef }} \mathrm{H}^{0}(X$, $\left.\mathcal{O}_{X}(N)\right)$.

Proof Let $A$ be an ample divisor on $X$ and let $\mathcal{G} \subset \operatorname{Cox}(X)$ be a set containing a non-zero section $s_{C}$ of $\mathrm{H}^{0}\left(X, \mathcal{O}_{X}(C)\right)$ for each integral curve $C$ with negative square and a generating set for $\bigoplus_{N \text { nef }} \mathrm{H}^{0}\left(X, \mathcal{O}_{X}(N)\right)$. We prove by induction on $n$ that for all divisors $D$ on $X$ with $A \cdot D=n$ the vector space $\mathrm{H}^{0}\left(X, \mathcal{O}_{X}(D)\right)$ is generated by monomials in $\mathcal{G}$. The result is clear if $n \leq 0$, since the only effective divisor $D$ with $A \cdot D \leq 0$ is the divisor $D=0$, and the vector space $\mathrm{H}^{0}\left(X, \mathcal{O}_{X}\right)$ is spanned by the empty product of the monomials in $\mathcal{G}$. Suppose that $n>0$ and that the result is true for all divisors $D^{\prime}$ such that $A \cdot D^{\prime}<n$. Let $D$ be a divisor on $X$ such that $A \cdot D=n$. If $D$ is either nef or not effective, then there is nothing to prove; so we reduce to the case in which $D$ is effective and not nef. Therefore there is an integral curve $C$ such that $D \cdot C<0$, and hence $C^{2}<0$ and $C$ is contained in the base locus of $|D|$. Thus the section $s_{C}$ divides all the vectors in $\mathrm{H}^{0}\left(X, \mathcal{O}_{X}(D)\right)$ and the result follows by the inductive hypothesis applied to the divisor $D-C$.

The following result is a consequence of [23, Proposition 3.3].

Proposition 2 Let $X$ be a smooth projective rational surface such that $-K_{X}$ is big. The cone of effective divisors on $X$ is finitely generated.

By Proposition 2, the semigroup of nef divisors on $X$ is finitely generated. To show that the "nef subring" of $\operatorname{Cox}(X)$ is finitely generated, it suffices to prove that every nef divisor on $X$ is semiample, by the following lemma of Zariski.

Lemma 1 ([17, Lemma 2.8]). Let $X$ be a projective variety and let $A_{1}, \ldots, A_{r}$ be semiample Cartier divisors on $X$. Then the ring

$$
\bigoplus_{\left(n_{1}, \ldots, n_{r}\right) \in \mathbb{Z}^{r}} \mathrm{H}^{0}\left(X, \mathcal{O}_{X}\left(n_{1} A_{1}+\cdots+n_{r} A_{r}\right)\right)
$$

is finitely generated.

The following sequence of lemmas shows that every nef divisor on $X$ is semiample, thus completing the ingredients necessary to prove Theorem 1 . The first lemma is well-known and holds on any smooth projective surface.

Lemma 2 Let $X$ be a smooth projective surface, let $N$ be a big and nef divisor, and let $C \subset X$ be an effective divisor such that $C \cdot N=0$. If $C_{1}, \ldots, C_{r}$ are distinct irreducible components of $C$, then the matrix $\left(C_{i} \cdot C_{j}\right)_{i, j}$ is negative definite, and $r \leq \operatorname{dim}\left(N_{1}(X)\right)-1$.

Proof Since $N$ is nef we have $N \cdot C_{i}=0$ for all $i$. Since $N$ is big and nef we have $N^{2}>0$ and the Hodge Index Theorem implies that the restriction of the intersection form to the span of $\left[C_{1}\right], \ldots,\left[C_{r}\right]$ is negative definite. Since the vectors $\left[C_{1}\right], \ldots,\left[C_{r}\right]$ are all orthogonal to $[N]$, to conclude it suffices to show that $\left[C_{1}\right], \ldots,\left[C_{r}\right]$ are linearly independent. Suppose that $a_{1}, \ldots, a_{r}$ and $b_{1}, \ldots, b_{r}$ are non-negative real numbers 
such that $a_{1} b_{1}=\cdots=a_{r} b_{r}=0$ and $\sum a_{i}\left[C_{i}\right]=\sum b_{j}\left[C_{j}\right]$. Thus the inequalities $0 \geq\left(\sum a_{i} C_{i}\right)^{2}=\sum a_{i} b_{j} C_{i} \cdot C_{j} \geq 0$ hold and we deduce that $\sum a_{i}\left[C_{i}\right]=0$. Since the surface $X$ is projective, we conclude that $a_{1}=\cdots=a_{r}=0$ and hence the matrix $\left(C_{i} \cdot C_{j}\right)_{1 \leq i, j \leq r}$ is negative definite.

Lemma 3 Let $N$ be a nef divisor on $X$ not linearly equivalent to zero.

1. We have $-K_{X} \cdot N>0$ and the linear system $|N|$ has dimension at least one.

2. If $C \subset X$ is an effective divisor such that $C \cdot N=0$, then the arithmetic genus of $C$ is non-positive; in particular every reduced connected component of $C$ has arithmetic genus zero and every integral component of $C$ is a smooth rational curve.

Proof (1) Write $-K_{X}=A+E$, where $A$ is an ample $\mathbb{Q}$-divisor and $E$ is an effective $\mathbb{Q}$-divisor. Since $N$ is nef, it is a limit of ample divisors and in particular it is in the closure of the effective cone. Because $N$ is not linearly equivalent to zero, Kleiman's ampleness criterion [19, Proposition IV.2.2] implies that $A \cdot N>0$, and hence

$$
-K_{X} \cdot N=A \cdot N+E \cdot N \geq A \cdot N>0
$$

since $N$ is nef and $E$ is effective. We conclude by applying the Riemann-Roch formula to the divisor $N$, together with Serre duality and the fact that $K_{X}-N$ is the opposite of a big divisor and is therefore not effective.

(2) Since $X$ is rational we have $\mathrm{H}^{1}\left(X, \mathcal{O}_{X}\right)=(0)$. From the exact sequence

$$
0 \longrightarrow \mathcal{O}_{X}(-C) \longrightarrow \mathcal{O}_{X} \longrightarrow \mathcal{O}_{C} \longrightarrow 0
$$

we deduce that $\mathrm{H}^{1}\left(C, \mathcal{O}_{C}\right)$ is contained in $\mathrm{H}^{2}\left(X, \mathcal{O}_{X}(-C)\right)$ and, by Serre duality, we have $\operatorname{dim} \mathrm{H}^{2}\left(X, \mathcal{O}_{X}(-C)\right)=\operatorname{dim} \mathrm{H}^{0}\left(X, \mathcal{O}_{X}\left(K_{X}+C\right)\right)$. By $(1)$ we have $-K_{X} \cdot N>0$ and by assumption $C \cdot N=0$; thus $\left(K_{X}+C\right) \cdot N<0$ which implies that $K_{X}+C$ is not effective, since $N$ is nef. It follows that the arithmetic genus of $C$ is non-positive.

Remark 1 Under the additional assumption that $-K_{X}$ is effective, it is possible to show that $h^{0}\left(X, \mathcal{O}_{X}(N)\right)=\left(N^{2}-K_{X} \cdot N\right) / 2+1$ : see [13, Theorem III.1 and Lemma II.2].

Lemma 4 If $N$ is a nef non big divisor on $X$, then $|N|$ is base point free.

Proof The result is clear if $N$ is trivial; hence we assume that $N$ is non-zero. By Lemma 3 (1) the linear system $|N|$ has dimension at least one and thus $N$ is linearly equivalent to a divisor $M+F$, where $M$ and $F$ are effective, and $M \neq 0$ is fixed component free; note that $M^{2}=0$ since $N$ is not big. Since $M+F$ is nef and not big, it follows that $M \cdot F=0$, and since $F$ is also not big we deduce that $0 \geq F^{2}=F \cdot(M+F) \geq 0$. Therefore the intersection pairing restricted to $M$ and $F$ is isotropic and we conclude by the Hodge Index Theorem that $M$ and $F$ are proportional. In particular the linear system $|N|$ is base point free, the number of its base points is at most $N^{2}=0$ and the lemma follows. 
Lemma 5 If $N$ is a big and nef divisor on $X$, then $N$ is semiample.

Proof Let $C \subset X$ denote the union of the integral curves orthogonal to $N$. By Lemmas 2 and 3 (2) the divisor $C$ satisfies the hypotheses of Artin's contractability criterion [2, Theorem 2.3] and therefore there exists a normal projective surface $X^{\prime}$ and a birational morphism $X \rightarrow X^{\prime}$ contracting only the connected components of $C$. By [2, Corollary 2.6] it follows that $N$ is linearly equivalent to a divisor whose support is disjoint from $C$, and therefore $N$ is the pull-back of a Cartier divisor $N^{\prime}$ on $X^{\prime}$. By the Nakai-Moishezon criterion, the divisor $N^{\prime}$ is ample and hence it is semiample. Thus its pull-back $N$ is semiample, as we wanted to show.

Proof of Theorem 1 By Proposition 2, the nef cone of $X$ is finitely generated. Lemmas 4, 5, and 1 together imply that the ring $\bigoplus_{N} \mathrm{H}^{0}\left(X, \mathcal{O}_{X}(N)\right)$, as $N$ ranges through all nef divisors, is finitely generated. By Proposition 2 there are only finitely many curves with negative self-intersection on $X$. Thus the result follows from Proposition 1.

Remark 2 We briefly explain why the surfaces of Examples 1 have big anticanonical class. This is clear for the surfaces of type (a). For a surface $X$ as in (b), let $\sigma$ and $F$ denote the inverse images of the negative curve and of a fiber, respectively, and let $\tilde{\sigma}$ and $F_{1}, \ldots, F_{n+1}$ denote the strict transforms of $\sigma$ and the special fibers, respectively. We may write

$$
-n K_{X}=(\sigma+n F)+\left((n-1) \sigma+n \tilde{\sigma}+n \sum F_{i}\right)
$$

which shows that $-n K_{X}$ is the sum of a big and an effective divisor, whence $-K_{X}$ is big. For a surface $X$ as in (c), let $p$ be the point of $\mathbb{P}^{2}$ not on the conic, let $c$ be the strict transform of the conic and let $\ell_{1}, \ldots, \ell_{n}$ be the strict transforms of the lines through $p$ and each one of the remaining blown-up points. We may write

$$
-n K_{X}=2 \ell+\left(\sum \ell_{i}+(n-1) c\right)
$$

which shows that $-n K_{X}$ is the sum of a big and an effective divisor, whence $-K_{X}$ is big. Similarly, for the surface (d), we note that $-2 K_{X}$ can be written as $\ell+E$ where $\ell$ is the inverse image of the class of a line in $\mathbb{P}^{2}$ (which is big) and $E$ is effective. For the surface (e) the divisor $-K_{X}$ is big by Sect. 4 .

\section{Big rational surfaces and log del Pezzo surfaces}

The following family of examples shows that there exist smooth rational surfaces $X$ with big anticanonical divisor which are not log del Pezzo surfaces. We show the stronger statement that there is no $\mathbb{Q}$-divisor $\Delta$ such that $(X, \Delta)$ is a log canonical pair and $-\left(K_{X}+\Delta\right)$ is ample.

Let $n \geq 2$ be an integer and let $h: \mathbb{F}_{n} \rightarrow \mathbb{P}^{1}$ be the Hirzebruch surface with a curve $\bar{\sigma}$ of square $-n$. Let $k$ be an integer such that $3 \leq k \leq n+1$ and let $a_{1}, \ldots, a_{k}$ be positive 
integers such that $\sum \frac{1}{a_{j}}<k-2$. Choose $k$ distinct integral curves $\bar{F}_{1}, \ldots, \bar{F}_{k} \subset \mathbb{F}_{n}$ contracted by $h$, and for $i \in\{1, \ldots, k\}$ choose $a_{i}$ distinct points $p_{1}^{i}, \ldots, p_{a_{i}}^{i}$ on $\bar{F}_{i} \backslash \bar{\sigma}$. Let $X$ be the blow-up of $\mathbb{F}_{n}$ along $\left\{p_{j}^{i} \mid 1 \leq i \leq k, 1 \leq j \leq a_{i}\right\}$; let

- $\sigma \subset X$ be the strict transform of the divisor $\bar{\sigma}$;

- $F_{i} \subset X$ be the strict transform of the divisor $\bar{F}_{i}$ for $i \in\{1, \ldots, k\}$.

We have $-K_{X}=2 \sigma+(n+2-k) F+\sum F_{i}$, where $F \subset X$ is the inverse image in $X$ of a fiber of the morphism $h$. Define $P$ and $N$ as follows

$$
\begin{aligned}
-K_{X}= & \overbrace{\frac{n+2-k}{n-\sum \frac{1}{a_{j}}} \sigma+(n+2-k) F+\sum_{i} \frac{n+2-k}{a_{i}\left(n-\sum \frac{1}{a_{j}}\right)} F_{i}}^{P} \\
& +\underbrace{\left(2-\frac{n+2-k}{n-\sum \frac{1}{a_{j}}}\right) \sigma+\sum_{i}\left(1-\frac{n+2-k}{a_{i}\left(n-\sum \frac{1}{a_{j}}\right)}\right)}_{N} F_{i} .
\end{aligned}
$$

Our assumptions on $n, k$ and $\sum \frac{1}{a_{j}}$ ensure that both $P$ and $N$ are effective. Since $P \cdot \sigma=P \cdot F_{i}=0$ and $P^{2}=\frac{(n+2-k)^{2}}{n-\sum \frac{1}{a_{j}}}>0$, it follows that $P$ is big and nef and thus $-K_{X}$ is big. Additionally $P \cdot N=0$, so by Lemma 2 the intersection matrix of the support of $N$ is negative definite and therefore $-K_{X}=P+N$ is the Zariski decomposition of $-K_{X}$.

By [25, Theorem 4.3], the morphism $f: X \rightarrow Y$ induced by $|P|$ is a $\log$ resolution of $Y:=\operatorname{Proj} \oplus_{m \geq 0}\left(\mathrm{H}^{0}\left(X, \mathcal{O}\left(-m K_{X}\right)\right)\right.$ and $f^{*}\left(-m K_{Y}\right)=m P$ for some $m \gg 0$. We claim that $(Y, 0)$ is not a log canonical pair. Indeed, note that

$$
K_{X}-f^{*}\left(K_{Y}\right)=-N
$$

and that $N$ is supported on divisors contracted by $f$. Thus, the pair $(Y, 0)$ is $\log$ canonical if and only if $2-\frac{n+2-k}{n-\sum \frac{1}{a_{j}}} \leq 1$, or equivalently, if and only if $\sum \frac{1}{a_{j}} \geq k-2$.

Next, we show that there is no $\mathbb{Q}$-divisor $\Delta$ such that $(X, \Delta)$ is a log canonical pair and $-\left(K_{X}+\Delta\right)$ is ample. To see this, note that if $-\left(K_{X}+\Delta\right)$ is ample then the divisor

$$
A:=-\left(K_{X}+\Delta\right)-f^{*}\left(-K_{Y}-f_{*}(\Delta)\right)
$$

which is supported on the exceptional locus of $f$, is $f$-ample. By [27, Lemma 7.1], it follows that all its coefficients are non-positive, and thus $-A$ is effective. Let $g: Z \rightarrow$ $X$ be a $\log$ resolution of $(X, \Delta)$, and let $g^{-1}(\Delta)$ be the strict transform of $\Delta$. We have

$$
\begin{aligned}
K_{Z}+g^{-1}(\Delta)-g^{*}\left(K_{X}+\Delta\right)= & \left(K_{Z}-g^{*} f^{*}\left(K_{Y}\right)\right)+\left(g^{-1}(\Delta)\right. \\
& \left.-g^{*} f^{*} f_{*}(\Delta)\right)-g^{*}(-A) .
\end{aligned}
$$


The coefficients of the divisors $g^{-1}(\Delta)-g^{*} f^{*} f_{*}(\Delta)$ and $-g^{*}(-A)$ are all negative. Since $(Y, 0)$ is not log canonical, there is a coefficient of $K_{Z}-g^{*} f^{*}\left(K_{Y}\right)$ which is strictly less than -1 , and thus $(X, \Delta)$ is not a log canonical pair.

Remark 3 Ivan Cheltsov pointed out that the minimal resolution of a $\mathbb{Q}$-factorial rational Fano surface with rational singularities is in fact an example of a smooth projective rational surface with big anticanonical divisor. This raises the question of whether the converse is also true.

\section{Blow-ups of the projective plane and root systems}

In this section we classify blow-ups $X$ of $\mathbb{P}^{2}$ at finite sets of points for which $-K_{X}$ is both big and effective. We do so by associating a root system to each big rational surface, extending a well-known construction for del Pezzo surfaces [22, Section IV.25].

Lemma 6 Let $X$ be a smooth projective surface and let $\mathcal{D}$ be a set of integral curves on $X$. There is a big divisor whose support is contained in $\mathcal{D}$ if and only if the lattice $\mathcal{D}^{\perp}$ is negative definite.

Proof $(\Leftarrow)$ Suppose that $\mathcal{D}^{\perp}$ is negative definite. By the Hodge Index Theorem, there is a divisor $B=\sum_{C \in \mathcal{D}} a_{C} C$ such that $B^{2}>0$. By Riemann-Roch and Serre duality, $h^{0}(m B)+h^{0}(K-m B)$ grows (at least) quadratically in $m$ and the same statement holds for $h^{0}(-m B)+h^{0}(K+m B)$. Since $(K-m B)+(K+m B)=$ $2 K$ it follows that $h^{0}(K-m B)$ and $h^{0}(K+m B)$ cannot both grow quadratically. We deduce that either $B$ or $-B$ is a big divisor and the result follows.

$\left(\Rightarrow\right.$ ) Suppose that $B=\sum_{C \in \mathcal{D}} a_{C} C$ is a big divisor with $a_{C} \in \mathbb{Z}$ for all $C \in \mathcal{D}$. Adding non-negative multiples of the curves in $\mathcal{D}$ to $B$ we reduce to the case in which $B$ is effective; thus the base locus of $B$ is supported on $\mathcal{D}$. It suffices to show that there exists a big and nef divisor $N$ in the integral span of $\mathcal{D}$, since then $N^{2}>0$ and $\mathcal{D}^{\perp} \subset N^{\perp}$ is negative definite by the Hodge Index Theorem. Choose $m \gg 0$ so that the moving part of $m B$ is big. Then, subtracting from $m B$ its base components we obtain the desired divisor $N$.

Lemma 7 Let $\Lambda$ be a negative definite lattice. The set

$$
R:=\left\{\alpha \in \Lambda \mid \alpha^{2} \in\{-1,-2\}\right\}
$$

is a root system in the span $E$ of $R$.

Proof We adapt the argument in [22, Section IV.25]. To check that $R$ is a root system in $E$ it suffices to verify the axioms in [18, Section III.9].

$\mathrm{R} 1$ The set $R$ is finite, does not contain 0 and spans $E$. This follows from the definition of $E$ and the fact that the pairing is definite.

$\mathrm{R} 2$ If $\alpha \in R$, then the only multiples of $\alpha$ in $R$ are $\pm \alpha$. If $m \in \mathbb{R}$ is such that $\alpha, m \alpha \in R$, then $m^{2} \alpha^{2} \in\{-1,-2\} \cap\left\{-m^{2},-2 m^{2}\right\}$ and $m$ is rational since $\alpha, m \alpha \in \Lambda$. We deduce that $m^{2}=1$. 
R3 If $\alpha \in R$, then the reflection $\sigma_{\alpha}$ fixing the hyperplane orthogonal to $\alpha$ leaves $R$ invariant. The reflection $\sigma_{\alpha}$ is given by

$$
\sigma_{\alpha}(x)=x-2 \frac{x \cdot \alpha}{\alpha \cdot \alpha} \alpha .
$$

It follows from the definitions that $\sigma_{\alpha}(x)^{2}=x^{2}$ for all $x \in \Lambda$.

R4 For every $\alpha, \beta \in R$ we have $\alpha \cdot \beta \in \mathbb{Z}$. This property holds for all vectors in $\Lambda$.

The lemma follows.

Let $X$ be a smooth projective surface and let $\alpha \in N_{1}(X)_{\mathbb{Z}}$. It follows from the adjunction formula that $\alpha^{2} \equiv K_{X} \cdot \alpha(\bmod 2)$. In particular, the quadratic form associated to any sublattice of $N_{1}(X)_{\mathbb{Z}}$ orthogonal to $K_{X}$ is even.

Proof of Theorem 2 If $\left|-K_{X}\right|$ contains an irreducible divisor $D$, then $D^{2}=9-r$ and, by Lemma 6, $D$ is big if and only if $r \leq 8$. Thus we reduce to the case in which every element of $\left|-K_{X}\right|$ is reducible and the set of blown-up points $\mathcal{P}$ is contained in the union of a line and a (possibly reducible) conic.

Suppose that $\mathcal{P}$ is contained in the union of a line $L \subset \mathbb{P}^{2}$ and an integral conic $C \subset \mathbb{P}^{2}$. Let $a$ be the number of points of $\mathcal{P}$ contained in $L \backslash C$ and let $b$ be the number of points of $\mathcal{P}$ contained in $C \backslash L$. If $a b=0$, then the result follows from Example 1 (c); thus we reduce to the case $a, b \geq 1$. Let $\ell \in \operatorname{Pic}(X)$ be the class of the inverse image of a line, let $e_{1}, \ldots, e_{a} \in \operatorname{Pic}(X)$ be the classes of the exceptional curves lying above the points of $\mathcal{P}$ in $L \backslash C$ and let $f_{1}, \ldots, f_{b} \in \operatorname{Pic}(X)$ be the classes of the exceptional curves lying above the points of $\mathcal{P}$ in $C \backslash L$. The divisor classes $e_{1}-e_{2}, e_{2}-e_{3}, \ldots, e_{a-1}-e_{a}, f_{1}-f_{2}, f_{2}-f_{3}, \ldots, f_{b-1}-f_{b}$, are orthogonal to the components of a general element of $\left|-K_{X}\right|$ and are positive roots of a root lattice of type $A_{a-1}(-1) \oplus A_{b-1}(-1)$. Therefore the intersection form restricted to the span of the above roots is negative definite. On the other hand, the vector $v:=a b \ell-b \sum e_{i}-2 a \sum f_{j}$ is orthogonal to the components of $-K_{X}$ and to the root lattice $A_{a-1}(-1) \oplus A_{b-1}(-1)$. It follows from Lemma 6 that $-K_{X}$ is big if and only if $v^{2}<0$; hence $-K_{X}$ is big if and only if $\frac{1}{a}+\frac{4}{b}>1$ and we conclude.

Suppose that $\mathcal{P}$ is contained in the union of three lines $L_{1}, L_{2}, L_{3} \subset \mathbb{P}^{2}$. For $i \in\{1,2,3\}$ let $a_{i}$ be the number of points of $\mathcal{P}$ contained in $L_{i}$ and not in $L_{j}$ for $j \neq i$, and let $e_{1}^{i}, \ldots, e_{a_{i}}^{i} \in \operatorname{Pic}(X)$ be the classes of the exceptional curves lying above such points of $\mathcal{P}$; let also $\ell \in \operatorname{Pic}(X)$ be the class of the inverse image of a line. If $a_{1} a_{2} a_{3}=0$, then the result follows from Example 1 (c); thus we reduce to the case $a_{1}, a_{2}, a_{3} \geq 1$. The divisor classes $\left\{e_{j}^{i}-e_{j+1}^{i} \mid i \in\{1,2,3\}, j \in\left\{1, \ldots, a_{i}-1\right\}\right\}$ are orthogonal to the components of the element of $\left|-K_{X}\right|$ whose image in $\mathbb{P}^{2}$ is $L_{1}+L_{2}+L_{3}$ and are positive roots of a root lattice of type $A_{a_{1}-1}(-1) \oplus A_{a_{2}-1}(-1) \oplus A_{a_{3}-1}(-1)$. Therefore the intersection form restricted to the span of the above roots is negative definite. The vector $v:=a_{1} a_{2} a_{3} \ell-a_{2} a_{3} \sum e_{i}^{1}-a_{1} a_{3} \sum e_{j}^{2}-a_{1} a_{2} \sum e_{k}^{3}$ is orthogonal to the components of $-K_{X}$ and to the root lattice $A_{a_{1}-1}(-1) \oplus A_{a_{2}-1}(-1) \oplus A_{a_{3}-1}(-1)$. It follows from Lemma 6 that $-K_{X}$ is big if and only if $v^{2}<0$; hence $-K_{X}$ is big if and only if $\frac{1}{a_{1}}+\frac{1}{a_{2}}+\frac{1}{a_{3}}>1$, and we conclude. 


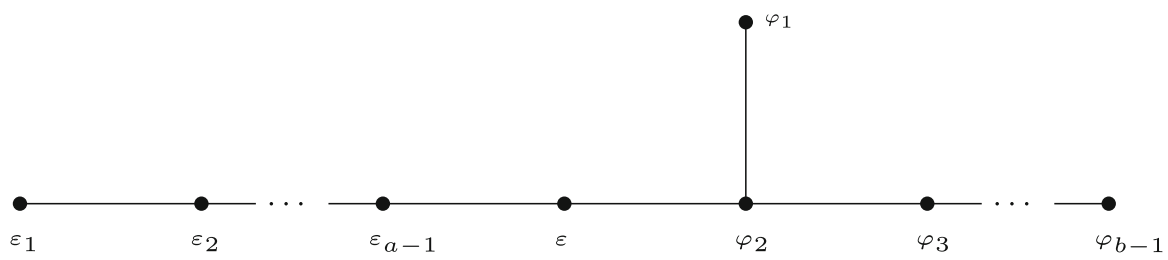

Fig. 2 The Coxeter graph for the root lattice $R_{a, b}$

Remark 4 We describe explicitly the root system of Lemma 7 contained in the lattice orthogonal to the components of an element of $\left|-K_{X}\right|$, when $X$ is one of the surfaces of Theorem 2.

For surfaces of type (1) we recover a subsystem of the usual root system associated to a del Pezzo surface [22, Section IV.25].

For surfaces of type (2), let $R_{a, b}(-1)$ be the orthogonal complement of the irreducible components of a reducible section of $-K_{X}$ of the kind mentioned in Theorem 2 . The lattice $R_{a, b}$ is spanned by a root system; a set of positive roots for $R_{a, b}$ is given by

$$
\begin{array}{ll}
\varepsilon_{i}:=e_{i}-e_{i+1} & \text { for } i \in\{1,2, \ldots, a-1\}, \\
\varphi_{j}:=f_{j}-f_{j+1} & \text { for } j \in\{1,2, \ldots, b-1\}, \\
\varepsilon:=\ell-e_{a}-f_{1}-f_{2} & \text { if } a \geq 1 \text { and } b \geq 2 .
\end{array}
$$

The associated Coxeter graph appears in Fig. 2.

The type of the root lattice $R_{a, b}$ varies with $a$ and $b$ : the following are the possibilities.

- If $a b=0$, then $R_{a, b}=A_{a+b-1}$.

- If $b=2$, then $R_{a, b}=A_{a}+A_{1}$.

- If $b=3$, then $R_{a, b}=A_{a+2}$.

- If $b=4$, or $a=1$ and $b \geq 4$, then $R_{a, b}=D_{a+b-1}$.

- If $a=2$ and $b=5$, then $R_{a, b}=E_{6}$.

- If $a=3$ and $b=5$ or $a=2$ and $b=6$, then $R_{a, b}=E_{7}$.

- If $a=4$ and $b=5$ or $a=2$ and $b=7$, then $R_{a, b}=E_{8}$.

Similarly, for surfaces of type (3), let $R_{a_{1}, a_{2}, a_{3}}$ be the opposite of the orthogonal complement of the components of $-K_{X}$. The lattice $R_{a_{1}, a_{2}, a_{3}}$ is a root system of type $A_{m}+A_{n}, D_{n}, E_{6}, E_{7}$ or $E_{8}$, depending on the values of $a_{1}, a_{2}$ and $a_{3}$. A set of positive roots for the root system is given by $\left\{\varepsilon_{j}^{i}:=e_{j}^{i}-e_{j+1}^{i} \mid i \in\{1,2,3\}, j \in\right.$ $\left.\left\{1, \ldots, a_{i}-1\right\}\right\}$, together with $\varepsilon:=\ell-e_{a_{1}}^{1}-e_{a_{2}}^{2}-e_{a_{3}}^{3}$ if $a_{1}, a_{2}, a_{3} \geq 1$.

Relabeling the indices if necessary we assume that $a_{1} \geq a_{2} \geq a_{3}$; note also that we necessarily have $a_{3} \leq 2$ and if $a_{3}=2$, then $a_{2} \leq 3$. The following are the possibilities.

- If $a_{3}=0$, then $R_{a_{1}, a_{2}, a_{3}}=A_{a_{1}-1}+A_{a_{2}-1}$.

- If $a_{3}=1$, then $R_{a_{1}, a_{2}, a_{3}}=A_{a_{1}+a_{2}-1}$.

- If $a_{2}=a_{3}=2$, then $R_{a_{1}, a_{2}, a_{3}}=D_{a_{1}+2}$.

- If $a_{2}=3$ and $a_{3}=2$, then $3 \leq a_{1} \leq 5$ and $R_{a_{1}, a_{2}, a_{3}}=E_{a_{1}+3}$ (Fig. 1). 


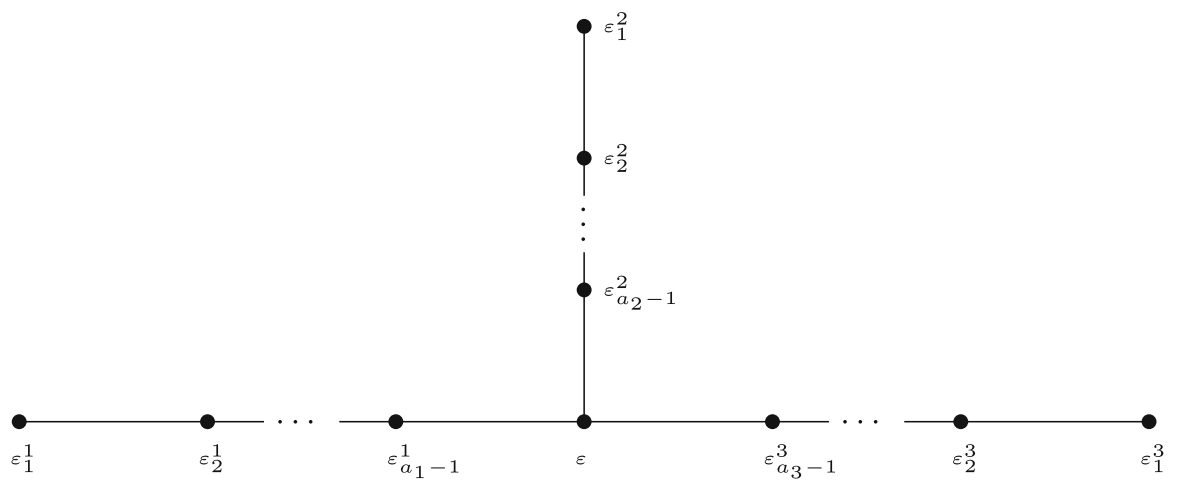

Fig. 3 The Coxeter graph for surfaces of type (3)

\section{The associated Coxeter graph appears in Fig. 3.}

Acknowledgments We thank Ana-Maria Castravet, Johan de Jong, David Eisenbud, Brian Harbourne, Brendan Hassett, Seán Keel, Bjorn Poonen, Burt Totaro and Chenyang Xu for helpful conversations during the completion of this work. We also thank the anonymous referee for a thorough report and helpful suggestions.

Open Access This article is distributed under the terms of the Creative Commons Attribution Noncommercial License which permits any noncommercial use, distribution, and reproduction in any medium, provided the original author(s) and source are credited.

\section{References}

1. Artebani, M., Laface, A.: Cox rings of surfaces and the anticanonical Iitaka dimension, 2009-10-5, Preprint arXiv:0909.1835v2 (math.AG)

2. Artin, M.: Some numerical criteria for contractability of curves on algebraic surfaces. Amer. J. Math. 84, 485-496 (1962)

3. Birkar, C., Cascini, P., Hacon, C.D., McKernan, J.: Existence of minimal models for varieties of log general type. J. Amer. Math. Soc. 23(2), 405-468 (2010)

4. Castravet, A.-M.: The Cox rings of Log-Fano surfaces (in preparation) (2008)

5. Chen, D., Schnell, C.: Electronic communication (2008-10-24)

6. Colliot-Thélène, J.-L., Sansuc, J.-J.: La descente sur les variétés rationnelles. II. Duke Math. J. 54(2), 375-492 (1987)

7. Cox, D.A.: The homogeneous coordinate ring of a toric variety. J. Algebraic Geom. 4(1), 17-50 (1995)

8. Du Val, P.: On the Kantor group of a set of points in a plane. Proc. Lond. Math. Soc., II. Ser. 42, 18-51 (1936)

9. Failla, G., Lahyane, M., Molica Bisci, G.: On the finite generation of the monoid of effective divisor classes on rational surfaces of type $(m, n)$, Atti della Accademia Peloritana dei Pericolanti Classe di Scienze Fisiche, Matematiche e Naturali, LXXXIV, 1-9 (2006)

10. Failla, G., Lahyane, M., Molica Bisci, G.: The finite generation of the monoid of effective divisor classes on Platonic rational surfaces. pp. 565-576. World Sci. Publ., Hackensack (2007)

11. Galindo, C., Monserrat, F.: The total coordinate ring of a smooth projective surface. J. Algebra 284(1), 91-101 (2005)

12. Harbourne, B.: Rational surfaces with $K^{2}>0$. Proc. Amer. Math. Soc. 124(3), 727-733 (1996)

13. Harbourne, B.: Anticanonical rational surfaces. Trans. Amer. Math. Soc. 349(3), 1191-1208 (1997)

14. Harbourne, B.: Electronic communication (2009-1-9)

15. Harbourne, B., Geramita, A.V., Migliore, J.: Classifying Hilbert functions of fat point subschemes in $\mathbb{P}^{2},(2008-8-8)$. Collect. Math. 60(2), 159-192 (2009) 
16. Hassett, B.: Rational surfaces over nonclosed fields, Arithmetic geometry, Clay Math. Proc., 8, pp. 155-209. Amer. Math. Soc., Providence (2009)

17. Hu, Y., Keel, S.: Mori dream spaces and GIT. Michigan Math. J. 48, 331-348 (2000)

18. Humphreys, J.E.: Introduction to Lie algebras and representation theory, Graduate Texts in Mathematics, 9, Springer, New York, Berlin, xii+171 (1978)

19. Kleiman, S.L.: Toward a numerical theory of ampleness. Ann. Math. (2) 84, 293-344 (1966)

20. Lazarsfeld, R.: Positivity in algebraic geometry. I. Ergebnisse der Mathematik und ihrer Grenzgebiete. 3. Folge., 48, Springer, Berlin, xviii+387 (2004)

21. Looijenga, E.: Rational surfaces with an anticanonical cycle. Ann. Math. (2) 114(2), 267-322 (1981)

22. Manin, Y.I.: Cubic forms: algebra, geometry, arithmetic. North-Holland Publishing Co., vii+292, Amsterdam (1974)

23. Nakayama, N.: Classification of log del Pezzo surfaces of index two. J. Math. Sci. Univ. Tokyo 14, 293-498 (2007)

24. Ottem, J.C.: On the Cox ring of $\mathbb{P}^{2}$ blown up in points on a line, to appear in Math. Scand. arXiv:0901.4277v5

25. Sakai, F.: Anticanonical models of rational surfaces. Math. Ann. 269(3), 389-410 (1984)

26. Totaro, B.: Hilbert's 14th problem over finite fields and a conjecture on the cone of curves. Compos. Math. 144(5), 1176-1198 (2008)

27. Zariski, O.: The theorem of Riemann-Roch for high multiples of an effective divisor on an algebraic surface. Ann. Math. (2) 76, 560-615 (1962) 